Volume 10, No.3, May - June 2021

International Journal of Advanced Trends in Computer Science and Engineering

Available Online at http://www.warse.org/IJATCSE/static/pdf/file/ijatcse1011032021.pdf

https://doi.org/10.30534/ijatcse/2021/1021032021

\title{
Lane Detection and Lane Departure Warning System
}

\author{
Sathyajith S ${ }^{1}$, Allwin Antony ${ }^{2}$, Jeevan C Paul ${ }^{3}$, Dinu Johnson ${ }^{4}$, Treesa Joseph ${ }^{5}$ \\ ${ }^{1}$ Department of Computer Science and Engineering, AISAT, Kalamassery, sathyajiths725@ gmail.com \\ ${ }^{2}$ Department of Computer Science and Engineering, AISAT, Kalamassery, allwinantony1999@ gmail.com \\ ${ }^{3}$ Department of Computer Science and Engineering, AISAT, Kalamassery, jeevanchelachodan @ gmail.com \\ ${ }^{4}$ Department of Computer Science and Engineering, AISAT, Kalamassery, dinujohnson726@ gmail.com \\ ${ }^{5}$ Department of Computer Science and Engineering, AISAT, Kalamassery, treesajoseph @ aisat.ac.in
}

\begin{abstract}
Lane detection is important for autonomous vehicles. For this reason, many approaches use lane boundary information to locate the vehicle inside the street, or to integrate GPS-based localization. Advanced driver assistance systems are developed to assist drivers in the driving process reducing road accidents. In this work, we present an end-to-end system for lane identification, clustering and classification, based on two cascaded neural networks, that runs in real-time. The first step is camera calibration which is used to remove the effect of lens distortion. Then a canny edge detection algorithm finds the edges of the images. Then the region of interest (ROI) is selected. The ROI is actually based on the rectangular shape appearing at the bottom of the image. ROI removes the unwanted region in the image. The potential lane markers are then determined using the Hough transform to analyze lane boundaries. Once the lane pixels are found, these pixels are continuously scanned to obtain the best linear regression analysis. It is qualified to be applied on highways and urban roadways. It also has been successfully verified in sunny, and rainy conditions for both day and night.
\end{abstract}

Key words : Canny edge detection, Hough transform, Lane departure warning System, Lane detection, Region of Interest.

\section{INTRODUCTION}

Traffic accidents have become one of the most serious problems in today's world. Roads are mostly the modes of transportation of choice and provide the best connections between all modes. The most frequently occurring traffic problem is driver's negligence and it becomes more serious with increase in Vehicles. Increasing human safety and saving their lives is the essential function of the Intelligent Transportation System (ITS). This system enables many tasks like, Users to be better informed and safer, more coordinated and smarter use of Transport networks. These road accidents can be reduced with the help of the road White lanes or signs that assists the driver in determining the road and non-road Area As traffic congestion increases, the number of casualties also increases. Driving safely under the condition of numerous vehicles and narrow roads has become the focus of attention. The perspective projection image is reworked into a corresponding bird's eye view followed by fitting parameters. Lane detection systems are useful in avoiding accidents, as safety is the main purpose of those systems they target to detect the lanes in all the conditions and classify the lanes. Drowsiness of drivers is one among the most common factors for road accidents. ADAS (Advanced Driver Assistance System) provides the automatic system to alter, enhance and adapt with the setting to cut back road fatalities and avoid collisions. The system either alerts the motive force or takes necessary actions of driving tasks providing necessary info like lane departure warning (LDW), position of vehicles, road boundary. One amongst the scientific fields Hough transform is applied on the ensuing binary image consisting of left and right halves. Finally, a matched filter involved in these tasks embraces Computer vision that performs tasks mechanically compared with human sensory system tasks. Computer vision senses the surroundings and might be applied in several applications supported by intelligent vehicle systems (ITS). In addition to the expressed explicit variations between the proposed methodology and also the existing approaches, it holds that the present approaches it holds that the present approaches largely use the HT for lane detection rather than the projected polynomial lane model that produces use of the similarity of lane markings. A notable exception is the add that develops a parallel snake lane model. completely different from the projected methodology, doesn't contemplate specializing in the additional reliable lane marking for lane detection.

\section{LITERATURE SURVEY}

Various approaches are mentioned for the lane detection system. Understanding of lane marking includes detective work, the structure of road, the position and variety of lanes, splitting, merging and ending lanes and roads in route road. abundant progress has been created in recent years. numerous sensing modalities like stereo sight, LIDAR, GPS, and has been used for higher improvement in understanding lanes A top-view transformation model has been planned for a vehicle parking assistant [3]. Here the Region of Interest (ROI) segmentation technique has been applied for intelligent road safety [4]. During this algorithmic program a top-hat transformation is being created as a preprocessing step for enhancing distinction of the image. The Hough transform has 
been used for lane detection. A vision based mostly lane departure detection technique has been developed [5] for urban roads. This system uses the Sobel operator. Then straight lanes are detected based on Hough transform estimating the lane dimension. A horizontal line detection technique has been given for detecting horizontal lines underneath that the lanes present [6]. This methodology uses a vertical mean distribution. Then a canny edge detector is applied marking edges of lanes and noise. Finally, K-means clustering and RANSAC algorithm has been applied for detecting lanes. Edges based mostly on lane detection methods. Edges based mostly on lane detection methodology have been analyzed step by step [7]. Few best-known and customary edge detection algorithms like Sobel, Canny, Roberts and Prewitt were analyzed and evaluated using image segmentation, morphology, image statistic and Hough transform. A superimposed approach has been provided for lane detection at midnight [8]. The lowest part as ROI is about eliminating the sky and alternative unsuitable objects. In any step the color channel is averaged to get greyscale. The dotted lines are elongated using temporal blurring. Then bright objects are extracted by threshold. Low resolution is iterated to extract lane markers. 3 modules-lane model generation, parameter estimation and matching has been developed for lane detection supported geometrical and Dennis Gabor filter [9]. Lane gradient maximized image has been created from a color image for lane detection in numerous illuminations [10].

\section{METHODOLOGY}

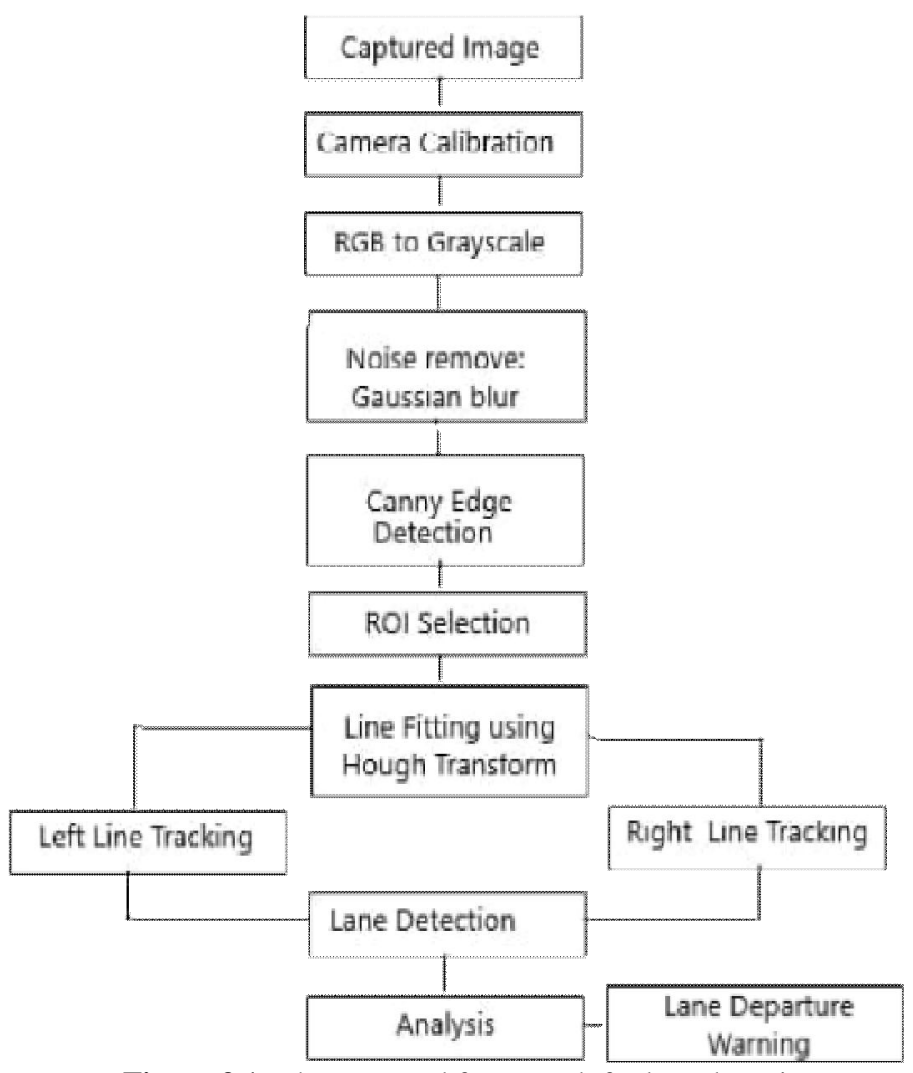

Figure 3.1: The proposed framework for lane detection

\subsection{Capturing image}

The first step involves capturing images. The input file may be a color image sequence captured by device. The camera is mounted simply at the front view mirror along the central line of the road within the vehicle. It captures images from the front view of a car together with road curvature, trees, buildings, shadows, vehicles so several objects don't return into account. The real time computer system hooked up with image capturing cards browse the pictures in real time and store them in memory. afterward the proposed system browses the image sequences stored in memory then begins the additional process steps. Few captured pictures from the main road throughout day time, sunny season as they are shown in "Fig 3.2, 3.3".

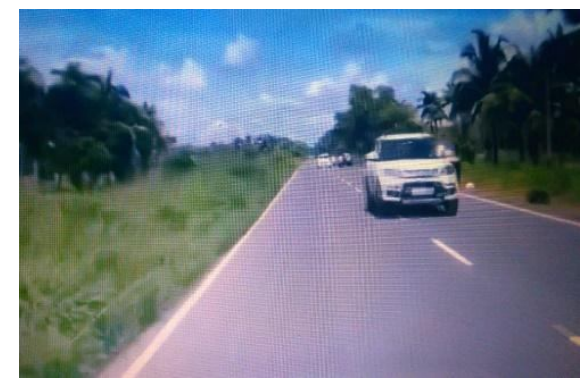

Figure 3.2: Captured image from highway road at day light

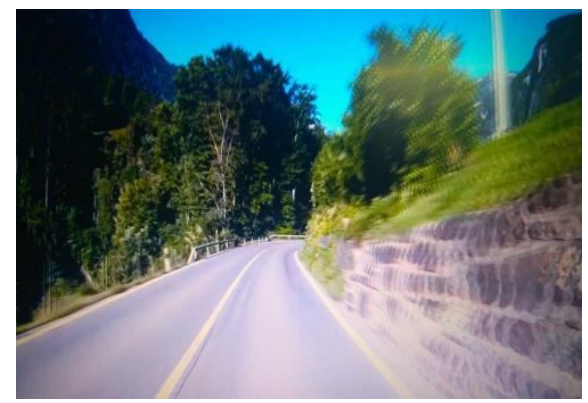

Figure 3.3: Captured image from highway road at sunny day

\subsection{Camera Calibration}

Camera calibration could also be needed to calibrate the captured image because of its distortion. Geometric camera calibration, conjointly noted as camera resectioning measures the parameters of the lens system and image sensing element of the captured image. To estimate camera parameters like intrinsic, extrinsic and distortion coefficients, 3D world organization points and their corresponding 2nd image organization square measure measured. To do this, a game board could be a best example as a multiple image of calibration pattern. The better-known approach to correct for that in the $(\mathrm{x}, \mathrm{y}, \mathrm{z})$ area is to apply coefficients to undistort the image. To calculate this a camera calibration method is needed. After camera activity a collection of functions are created to figure on check pictures before later getting used in a very video pipeline. Once we've a bird's eye view with a 
combined threshold we tend to be during a position to spot lines and a polynomial to draw a line.

$$
P=\left[\begin{array}{c}
R \\
t
\end{array}\right] k
$$

The optical center of the camera points to the origin of the camera's frame of reference that contains the rotation $\mathrm{R}$ and translation $\mathrm{t}$. but the intrinsic matrix $\mathrm{K}$ is calculated to support its focal distance, principal length additionally referred to as optical center and skew constant.

\subsection{RGB to Grayscale Conversion}

To retain the colour info and section the road from the lane boundaries exploiting the colour info faced difficulties on edge detection. In observation the paved surface may be created from many alternative colours because of shadows, completely different pavement vogue or age, that causes the colour of the paved surface and lane markings to alter from one image region to a different. Therefore, color images are regenerated into grayscale. However, the process of grayscale pictures becomes less as compared to a color image.

This operation transforms a 24-bit, three-channel, color image to associate degree 8bit. Segmentation of lane boundaries from the road surface using the colour data becomes troublesome for edge detection. The gradient of the road surface and lane markings change invariantly because of its shadows, buildings, trees and invariant illumination. pictures are made of picture elements and every pixel within the image may be a combination of three intensities. Thus pictures are regenerated into grayscale which means that three channel color image consisting of red, green and blue channel-24 bit is currently regenerated to at least one channel grey 8 bit. The purpose being by employing a grayscale image process, one channel is quicker than processing a three channel color image and less process intensive.

$$
\text { Grayscale }=(\mathrm{R}+\mathrm{G}+\mathrm{B}) / 3
$$

You may encounter an uint8 overflow error - the add of R, $\mathrm{G}$, and $\mathrm{B}$ is larger than 255. To avoid the exception, R, G, and $\mathrm{B}$ ought to be calculated severally.

The average technique is straightforward but doesn't work likewise obviously. the explanation being that human eyeballs react differently to RGB. Eyes are most sensitive to inexperienced light, less sensitive to red light, and therefore the least sensitive to blue light. Therefore, the 3 colours ought to have completely different weights within the distribution. That brings us to the weighted technique. The weighted technique, additionally known as luminousness technique, weighs red, inexperienced and blue consistent with their wavelengths. The improved formula is as follows:

$$
\text { Grayscale }=0.299 \mathrm{R}+0.587 \mathrm{G}+0.114 \mathrm{~B}
$$

\subsection{Edge Detection}

Edges are the points where the gray scale values modify sharply. Edge detection could be an important step in deciding the location of lane boundaries because the edge is one amongst the most important info contributors to the lane. The biggest issue of edge detection is the detection of the precise edges together with the great orientation of the lane boundary within the image. as a result of the memory needed to store the sides of the image is a smaller amount compared to the full image view. but it contains all info of the lane boundary and additionally orientation of the lane. In our stage method as shown in "Fig 3.4, 3.5". The strategy is predicated on the characteristics of intensity values of considered components. A proof to noise ratio (SNR) on the sting detector could also be applied so edges may well be found even if the data quality is poor. Gradient is the amendment in brightness or separation over a series of pixels. So, the canny edge computes the gradient of the blurred image and so traces the strongest gradients as a series of white pixels. Lane boundaries are outlined by sharp contrast between the road surface and painted lines or some form of non-pavement surface. These sharp contrasts are edges within the image Therefore edge detectors are vital in deciding the situation of lane boundaries. It conjointly reduces the quantity of learning knowledge needed by simplifying the image significantly, if the definition of a road may be extracted from the image. The edge detector was enforced for this formula and therefore the one that made the edge pictures was the 'canny' edge detector.

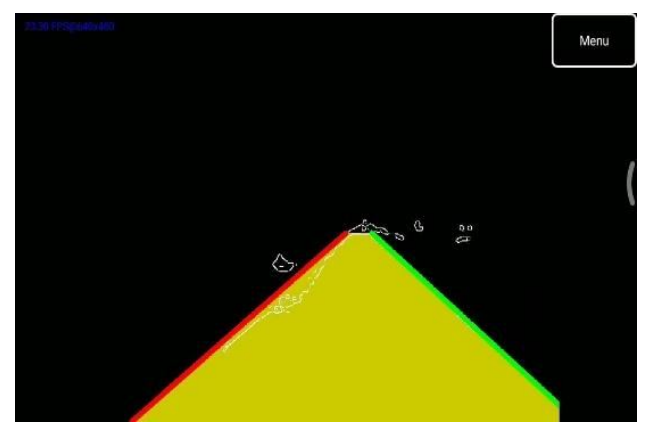

Figure 3.4: Image of edge detection on a highway road

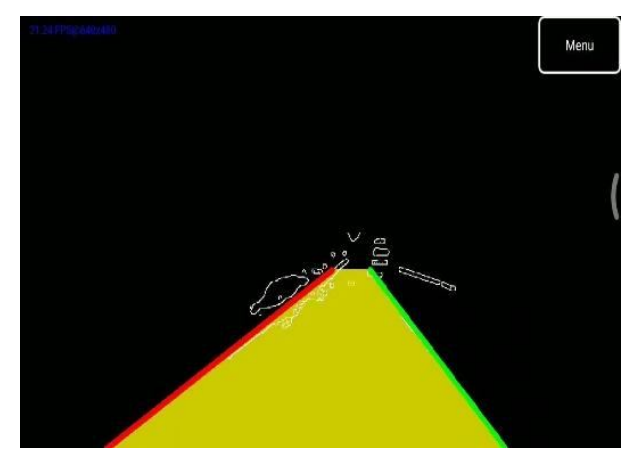

Figure 3.5: Image of edge detection on a non-highway road 


\subsection{Region of Interest}

In this step lane lines are detected using ROI that excludes the other extraneous objects. There can be many objects within the image that don't seem to be of interest like trees, buildings, humans etc. A ROI is isolated based on the region of interest that traces a Rectangle limiting the extent view. ROI defines the border of the lanes that includes individual points of interest. Here the canny image is disguised with the mask image to trace the ROI Rectangle shape by bitwise and operator. Thereby masking the whole region, the operation happens component wise, therefore all the white pixels within the region are operated against the corresponding region of the smart image. So, by bitwise and operation which implies with success disguised our smart image to ultimately show the ROI the region copied by the plane figure contour in the gray image, most of the image data is useless for lane detection. It will scale back the procedure quality of the formula by initializing a useful region of interest (ROI) of lane detection. The format of ROI is illustrated in "Fig.3.6, 3.7". After edge detection by canny edge detection, we will see that the obtained edge does not solely include the specified lane line edges system; we have a tendency to apply canny edge detection, a regular and common edge detection algorithm. The smart edge detector works during a multi edges, however conjointly includes alternative excess lanes and also the edges of the encompassing fences. The way to take away these additional edges is to see the space cortical region \} of a parallelogram and solely leave the edge info of the visible area. The premise is that the camera is mounted relative to the car, and also the relative position of the car with relevancy the lane is additionally mounted, in order that the lane is basically kept in a very mounted space within the camera.

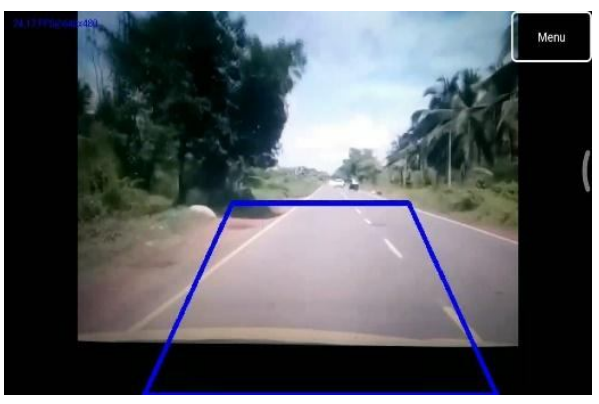

Figure 3.6: Region of Interest

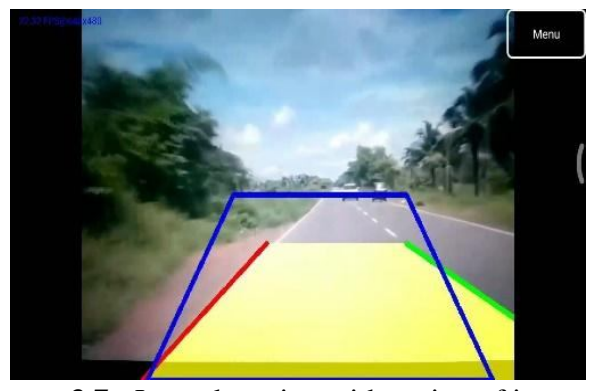

Figure 3.7: Lane detection with region of interest

\subsection{Hough Transform}

This step involves using Hough transform technique to find straight lines in our region of interest and so determine the lane lines. This method uses a constant quantity area known as Hough area. Hough transform may be a mechanism that provides additional weightage to pixels that are already in line by an option procedure. This option procedure is disbursed during a parameter area from which lines are obtained as native maxima. The lines that are outside the area are rejected as shown in "Fig 3.8". For instance, any horizontal line that's not a part of the lane boundary will be neglected further.

Hough transform halves the image is splitted in yielding left and right aspect of the image Hough remodel may be a technique that may be used to record vote for every cast of votes within the bin that it belongs to with the utmost variety of votes Threshold is that the minimum variety of intersections to find a line antecedently mentioned during a series of points.

Hough area represents the letter and alphabetic character values of lines that are common between a series of points. for each purpose of intersection of vote within the bin it belongs to a line with some value of alphabetic character and letter. Finally, lines are detected from a series of points within the gradient image exploitation Hough transform and these lines are shown within the original color image by mixing the lines with the initial image.

The algorithm has been modified and increased to be ready to acknowledge different shapes like circles and quadrilaterals of specific sorts. So as to know how the Hough transform algorithm works, it's vital to know four concepts: edge image, the Hough space and also the mapping of edge points onto the Hough space, associate degree alternate way to represent a line, and the way lines are detected. For the Hough transform algorithm, it's crucial to perform edge detection initially to supply an edge image which is able to then be used as input into the algorithm regardless of the values of theta, the line is drawn since it absolutely was voted because the line of best match scribing the information supported the threshold.

Threshold is the minimum variety of intersections to notice a line previously mentioned in a very series of points. Hough space represents the theta and rho values of lines that are common between a series of points. for each purpose of intersection of vote within the bin it belongs to a line with some worth of rho and theta.

Finally, lines are detected from a series of points within the gradient image victimization Hough transform and these lines are shown within the original color image by mixing the lines with the first image purpose ie. to seem for lines that get several votes. These purposes of intersections are within a bin of Hough area for each point of intersection . 


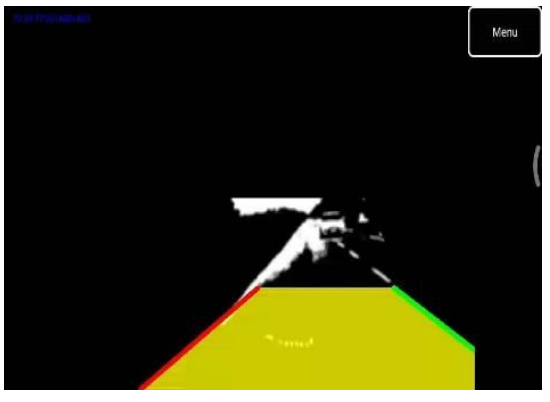

Figure 3.8: Hough transform with lane detection

\subsection{Finding Lane Line}

Multiple lines with completely different slopes and length are formed using Hough transform. The final word resolution is to have solely 2 lines, one for right and alternative for left lane. And it's necessary that the lines depending on either facet are all roughly more or less getting to a similar direction. Left line includes a negative slope and the right one includes a positive slope Averaging out the slope and y intercept of multiple lines into one line traces out each of the correct and left lane. Left fit contains the averaged lines on the left and intuitively right fits containing coordinates of the road at right. Thus, the lines are getting to have similar vertical coordinates and are going to begin at rock bottom and go upward. Hough lines vary long, therefore the most significant and applicable lines are those that are longest in length. Any detected line copied by less than minimum line length is going to be rejected. Since shorter lines could also be because of the noise or different random calculations. The lane detection module is principally divided into lane edge detection and linear lane detection. This section implements the essential functions of lane detection and performs lane detection based on improved preprocessing and also the planned ROI choice as shown in "Fig. 3.9, 3.10".

The ways of lane detection include feature primarily based methods and model-based methods. The tactic's primarily based feature is employed during this paper to notice the color and edge features of lanes so as to enhance the accuracy and potency of lane detection.

There are 2 ways to realize straight lane detection. One is to use the Hough line detected to perform encapsulation by the OpenCV library usually used for image processing, and draw lane lines within the corresponding space of the initial image. The opposite is self-programming. Within the header file, the ROI space is traversed to perform line detection for a particular variety of angles each way will be mirrored within the video, and therefore the initial methodology runs quicker.

Since this text focuses on the accuracy and potency of lane detection, we tend to select the primary methodology (Hough line duty within the OpenCV library to run quicker for linear detection. Moreover, as a result of the Hough rework is insensitive to noise and might process straight lines well, Hough transform is employed to extract lane line parameters in every frame of the image sequence for lane detection. In the image process, the Hough transform is employed to notice any form which will be expressed in an exceedingly mathematical formula, even though the form is broken or somewhat distorted. Compared with different ways, the Hough rework will realize noise reduction higher than different ways. In the image process, the Hough transform is employed to notice any form which will be expressed in an exceedingly mathematical formula, even though the form is broken or somewhat distorted. Compared with different ways, the Hough rework will realize noise reduction higher than different ways.

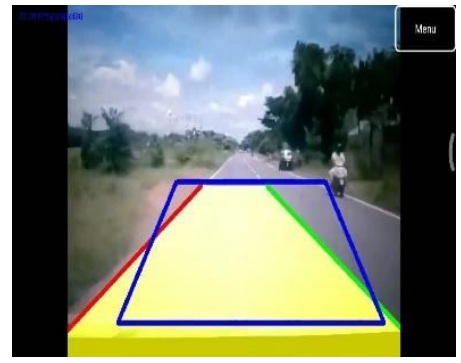

Figure 3.9: Lane detection with Region of interest

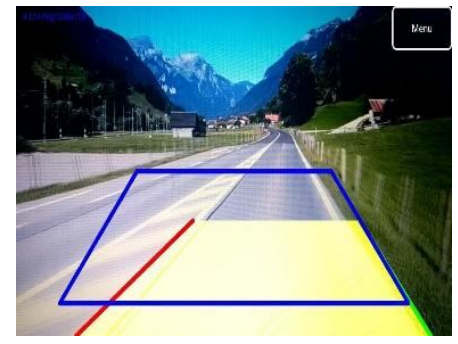

Figure 3.10: Lane detection with Region of interest from a different perspective.

\subsection{Warning}

An Alarm is going to be triggered whereas the vehicle deviates from the lane. With the assistance of each Hough transform and canny edge Detection Lane are going to be detected that comes within the region of Interest (ROI). An alarm can alert the system once it changes the lane with tone This feature depends on route markings to operate. It's designed to provide you with a warning if your car begins to drift out of a lane with one or additional kinds of warnings.

As you drive, continuously remember of your surroundings and also the traffic within the lanes beside you. However, if you accidentally begin to drift out of your lane, use this feature to assist keep you among your lane. If you are doing drift out of your lane, you'll receive warnings on your dashboard, hear a sound, this feature depends on lane markings to operate. This feature isn't designed to figure on 
unpaved roads or roads without lane markings as shown in "Fig.3.11, 3.12".

Lane keeping assist could be a feature that, in addition to the lane departure warning system, mechanically takes steps to confirm the vehicle stays in its lane. Some vehicles mix adjustive controller with lane keeping systems to supply further safety. whereas the mixture of those options creates a semi-autonomous vehicle, most need the driving force to stay on top of things of the vehicle whereas it's in use. This is often as a result of the restrictions related to the lane-keeping feature. The lane keeping assist system is being achieved in modern vehicle systems mistreatment image process techniques referred to as Hough transform and canny edge detection techniques.

These advanced image process techniques derive lane data from forward facing cameras connected to the front of the vehicle. A lane keeping assist mechanism will either reactively turn a vehicle into the lane if it starts to go away or proactively keep the vehicle within the center of the lane.

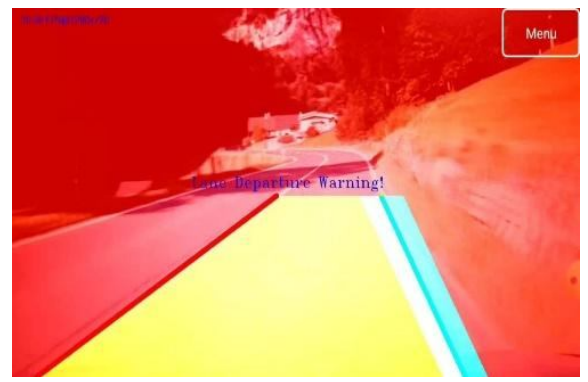

Figure 3.11: Lane Departure Warning

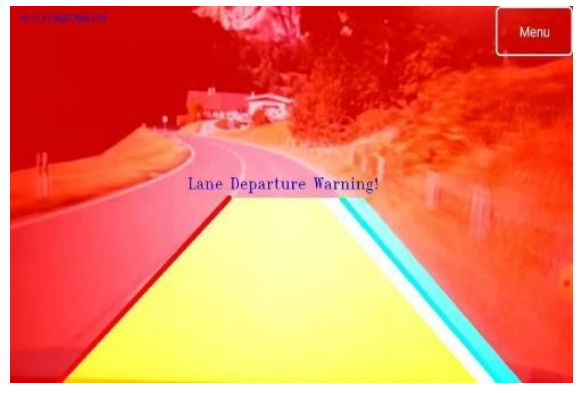

Figure 3.12: Lane Departure Warning from a different angle

\section{RESULT}

The experiment is performed on moving vehicles shooting in main roads underneath numerous illuminations with totally different distances starting from 10-60 meters. The camera height from the bottom should be an exact worth that's less than two meters. The captured pictures from totally different distances and therefore the detected resultant pictures are shown in "Fig.4.1, 4.2, 4.3" The blue lines within the pictures show the results of the final process of lane detection. As discovered from the figures, the lanes are simply detected at distances and different illuminations with such a large amount of noise. The results reveal that the planned system isn't tormented by the surroundings of the streets; have the ROI selected appropriate for any set detection of the lanes underneath the significant rain. the general performance of the planned system is performed qualitatively and quantitatively. This analysis ranges in main roads throughout day, sunny and night. In order to generalize the lane detection, we tend to get the image whereas driving on an specific main road or a national road at $80-100 \mathrm{~km} / \mathrm{h}$ while not considering any constraint of a light-weight, shadow, moving vehicle etc. We tend to create the experiment considering a numerous of road and a road condition within the manner of dependability check for the road detection. The figures show the results recognizing a road line or boundary through the proposed algorithm and conjointly shows the line detection on a curved road. It conjointly shows the results of lane recognition just in case of an outsized curvature of a road and also, we will see the right line recognition underneath the shadow of trees and a vehicle, or piecewise lane, and see quick process performance of 20 frame/sec over.

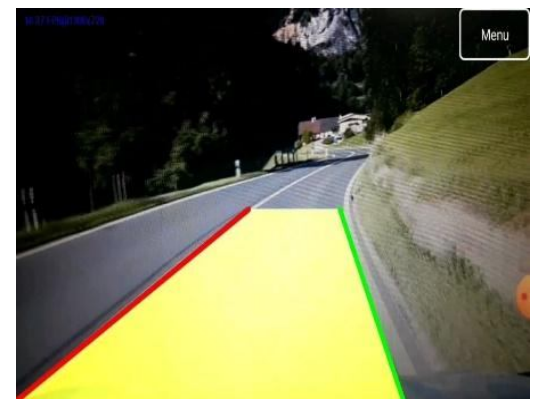

Figure 4.1: Final lane detection

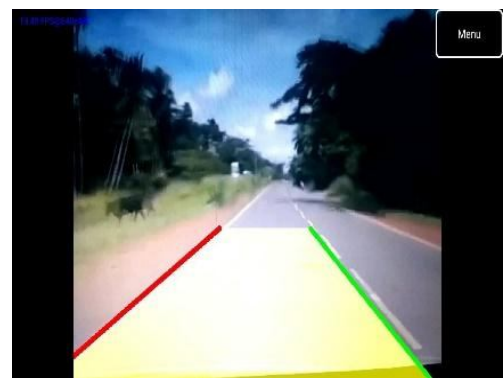

Figure 4.2: Final lane detection from a different angle

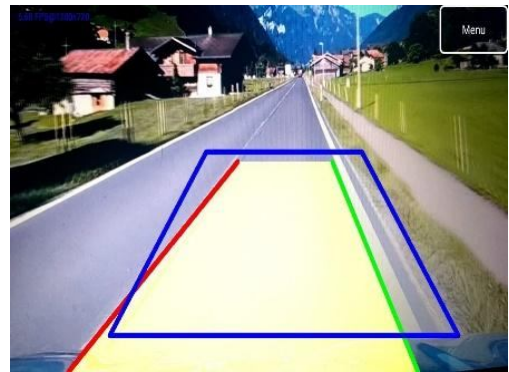

Figure 4.3 : Final lane detection when region of interest enabled 


\section{CONCLUSION}

This paper proposes a robust lane detection technique beneath the idea that lane markings are parallel. Experiments show that sleuthing the additional reliable lane marking and estimating the opposite one performs fine even in complicated conditions. This led to our presentation of the ROI based mostly lane-tracking system operated in a very wide selection of environments. For frames recorded by the camera. However, some downsides weren't solved, like sharp curves within the foreground of the image and therefore the correct safe lane systems, this approach developed a security system for avoiding lane departure for an outsized and complicated set of traffic situations. According to our experiment results of the projected methodology during this paper, we are able to conclude that this technique may be used beneath most of route road in daylight, night time, sunny so on. Additionally, it may be used beneath numerous - straight lines still like dash lines, lane markings and vehicles for lane boundary recognition. supported ROI and Hough transform voting system, the system will acknowledge lane boundaries by means of mobile vision and forward collision functions. grey scale data point, ROI, Hough transform-voting based mostly approaches square measure applied to acknowledge the lane boundaries. To alter the installation step, the systems are virtually adjustive to most of the vehicles. A mobile lane departure warning system is nearly a commercialised product that brings driving safety to the drivers and passengers. Moreover, mobile lane departure warning system may be a Windows-based program that the users as long as transfer it to their own good phones and revel in the service. Within the future, the preceding vehicle detection and distance estimation algorithmic rule are integrated into the mobile lane departure warning system to be a crash dodging system. which will be a challenge to mix these functions within the restricted computing resource on good phones. To reduce the high computational value, the image is reduced to a smaller region of interest. Smaller threshold value is applied on edge detection for a lot of advanced environments.

\section{LIMITATION AND FUTURE STUDIES}

Lane departure warning systems and lane keeping systems accept visible lane markings. They generally cannot decipher light, missing, or incorrect lane markings. Markings lined in snow or flooded lane markings left visible will hinder the flexibility of the system. limitations of road things give a major improvement to accuracy of detection of lane markers. Knowing the limitation of the system allows the system to figure in higher ways and reduce warning. The future scope of the lane detection includes a complicated atmosphere taking under consideration the various environments like the Weather conditions: fog, mist, cloudy, sunny, bright day light, darker, shadow or once there happens obstacles and Humps, Speed Breakers within the Road.

\section{REFERENCES}

1. Ammu M Kumar and Philomina Simon, Review Of Lane Detection And Tracking Algorithms In Advanced Driver Assistance System, International Journal of Computer Science \& Information Technology (IJCSIT) Vol 7, No 4, August 2015.

2. Aharon Bar Hillel · Ronen Lerner - Dan Levi · Guy Raz, Recent progress in road and lane detection: a survey, Machine Vision and Applications (2014) 25:727-745 DOI 10.1007/s00138-011-0404-2, Received: 28 October 2010 / Revised: 12 December 2011 / Accepted: 19 December 2011 / Published online: 7 February 2012 () Springer-Verlag 2012.

3. Chian-Chuan Lin, and Ming-Shi Wang,A vision Based Top-View Transformation Model for a Vehicle Parking Assistant, Sensors 2012,12,44314446,doi:10.3390/s120404431,ISSN 14248220,www.mdpi.om/journal/sensors,30 March 2012.

4. Prof. Sachin Sharma and Dr. D. J. Shah, A Much Advanced and Efficient Lane Detection Algorithm for Intelligent Highway Safety, Sundarapandian et al. (Eds) : ITCS, SIP, CS \& IT 09, pp. 51-59, 2013. () CS \& IT-CSCP 2013, DOI : 10.5121/csit.2013.3106.

5. Y.-C. Leng and C.-L. Chen, Vision-based lane departure detection system in urban traffic scenes, in Control Automation Robotics Vision (ICARCV ), 2010 11th International Conference on , 2010, pp. 1875- 1880.

6. T.T. Tran, H.M.Cho, S.B.Cho, A robust method for detecting lane boundary in challenging scenes. Information Technology Journal. Image Process., vol. 10, no.12, pp. 2300-2307, 2011.

7. Moveh Samuel, Maziah Mohamad, Shaharil Mad Saad, Mohamed Hussein,Development of Edge-Based Lane Detection Algorithm Using Image Processing, International Journal On Informatics Visualization, Vol 2 (2018) No 1 e-ISSN : 2549-9904 ISSN : 2549-9610.

8. A.Borkar, M. Hayes, M. Smith, and S. Pankanti, A layered approach to robust lane detection at night, in Proc. IEEE Workshop Comput. Intell. Vehicles Veh. Syst., 2009, pp. 51-57.

9. S.Zhou, Y.Jiang, J. Xi, J. Gong, G. Xiong, and H. Chen, A novel lane detection based on geometrical model and gabor filter, in Intelligent Vehicles Symposium (IV ), 2010 IEEE , June 2010, pp. 59-64. www.powerdivision.gov.bd/user/brec1/30/1, accessed on August 29, (2015).

10. H.Yoo, U.Yang, and K. Sohn, Gradient-enhancing conversion for illumination-robust lane detection, IEEE Transactions on Intelligent Transportation Systems, vol. 14, pp. 1083 -1094, September 2013. [11]Shweta N. Dethe, Varsha S. Shevatkar, Prof. R. P. Bijwe," Google driverless car",IJSRSET | Volume 2 | Issue 2 | Print ISSN : 2395-1990 | Online ISSN : 23944099,2016. 